\title{
DEVELOPMENT AND VALIDATION OF COUPLED DYNAMICS CODE 'TRIKIN' FOR VVER REACTORS
}

\author{
OBAIDURRAHMAN K. ${ }^{*}$, J. B. DOSHI ${ }^{1}$, R. P. JAIN ${ }^{1}$, and V. JAGANNATHAN ${ }^{2}$ \\ Atomic Energy Regulatory Board, Anushaktinagar, Mumbai, India 400094 \\ ${ }^{1}$ Department of Mechanical Engineering, IIT Bombay, Mumbai, India 400076 \\ ${ }^{2}$ Light Water Reactors Physics Section, Bhabha Atomic Research Centre, Mumbai, India 400085 \\ *Corresponding author. E-mail : obaid@aerb.gov.in and obaid.iitb@gmail.com
}

Received June 03, 2009

Accepted for Publication April 29, 2010

New generation nuclear reactors are designed using advanced safety analysis methods. A thorough understanding of different interacting physical phenomena is necessary to avoid underestimation and overestimation of consequences of offnormal transients in the reactor safety analysis results. This feature requires a multiphysics reactor simulation model. In this context, a coupled dynamics model based on a multiphysics formulation is developed indigenously for the transient analysis of large pressurized VVER reactors. Major simplifications are employed in the model by making several assumptions based on the physics of individual phenomenon. Space and time grids are optimized to minimize the computational bulk. The capability of the model is demonstrated by solving a series of international (AER) benchmark problems for VVER reactors. The developed model was used to analyze a number of reactivity transients that are likely to occur in VVER reactors.

KEYWORDS : Space-time Kinetics, IQS Method, Coupled Analysis, VVER Reactors, Thermal Hydraulics, Safety Analysis

\section{INTRODUCTION}

Reliable transient analysis of nuclear reactors is very important to understand the consequences of different events on nuclear safety. A reliable analysis approach is one that can address the finer details of all interacting physical phenomena. Traditionally, the thermal hydraulics (TH) codes and nuclear kinetics codes were developed independently to pursue different objectives and had little or no common connections. Such an independent analysis approach might lead to substantial ambiguity in the understanding of core physics and thermal hydraulic phenomenon. Thus, the use of advanced computational methods, capable of reflecting the real physics of the systems, is essential. Of major interest to nuclear engineers is the use of best estimate methods that can predict important safety margins and their associated uncertainties. The development of coupled codes and combining thermal hydraulic system codes and 3D neutron kinetics codes is an important step to perform best estimate calculations for plant transients. In the present age, with recent computer developments resulting in the availability of powerful computation capabilities at reasonable costs, addressing the interconnection between the two physical phenomena has become quite feasible. This type of detailed overall $\mathrm{TH} /$ neutronics simulation capabilities of transients in the nuclear industry will provide a basis for undertaking more in-depth evaluations of the safety margins found in earlier simulations for which a point kinetics model or a 1D model was used. Consistent with this objective, a number of projects to upgrade the quality of the safety analyses have been undertaken by the nuclear industry. During the last few years, different code systems, such as BIPR8, DYN3D, KIKO-3D, NEM, PARCS, and so on, have been implemented [1].

Generally, 3D neutron kinetics models of these codes use nodal expansion methods to solve two group neutron diffusion equations; the thermal hydraulic parts consist of fuel rod models describing fuel and coolant dynamics. The thermal hydraulic simulation of the core is intended for reactivity feedback purposes and to determine the core safety limits such as fuel enthalpy, MDNBR, etc.

A new computer code, named 'TRIKIN', (Triangular meshes based kinetics code with TH feedback) has been developed by the Indian Atomic Energy Regulatory Board to perform detailed safety analyses of the large VVER reactors that have been inducted into the Indian nuclear program. The TRIKIN model employs proven methodologies of the existing kinetics code NEUT [2] and the core physics code TRIHEX-FA [3] for VVER systems. The TRIKIN model also includes a simple multichannel thermal hydraulics module to address reactivity feedback and determine the thermal safety parameters. Some salient features of the code are discussed in Section 2. 


\section{SALIENT FEATURES OF TRIKIN CODE}

TRIKIN is a kinetics code based on a flux factorization technique employed by the improved quasistatic (IQS) method. The core physics calculations are based on the finite differencing scheme of the TRIHEX-FA code [3]. Stiffness related problems of kinetics equations are tackled efficiently by employing the generalized Runge-Kutta (GRK) method [4]. The core thermal hydraulics is modeled by an equivalent multichannel representation using a lumped 1D model. The mass, momentum and energy conservation equations are solved using proven semiimplicit computational schemes. The numerical grid is optimized based on the stability, convergence and accuracy requirements of individual physical phenomenon. The main features of TRIKIN can be summarized as follows.

- IQS factorization.

- Two/five neutron energy group provision.

- Every hexagonal fuel assembly is further divided into a number of triangular meshes and many axial layers.

- Flux is assumed to be uniform in every triangular mesh.

- Provision is available to eliminate bulky reflector regions from the computational domain using an appropriate albedo boundary condition.

- Stiff kinetic equations are handled using the GRK technique.

- One dimensional coolant dynamics module.

- Computational grid is optimized to fulfill the accuracy requirements of each physical phenomenon independently.

- Rapid evaluation of safety parameters such as MDNBR, clad temperature limits, etc.

- An approximate model for rapid evaluation of void fractions under phase change situations without detailed two phase equations.

\section{SPACE-TIME KINETICS MODEL}

The starting point for describing reactor transients is the time dependent, multi-group, multi-dimensional neutron diffusion equation, which is a simplified form of the Boltzmann transport equation, along with the associated equation for delayed neutron precursors. At any time $t$ and space point $r$, the time dependence of the neutron flux $\phi_{g}$ of the energy group g can be written as:

$$
\begin{gathered}
\frac{1}{v_{g}} \frac{\partial \phi_{g}}{\partial t}=\nabla \cdot D_{g} \nabla \phi_{g}(r, t)-\sum_{g}^{r} \phi_{g}(r, t)+\chi_{g}^{p}(1-\beta) \sum_{\substack{g \\
g}} \sum_{g}^{f} \phi_{g}(r, t) \\
+\sum_{g \neq g} \sum_{g^{\prime} \rightarrow g} \phi_{g}(r, t)+\chi_{g}^{d} \sum_{i} \lambda_{i} C_{i}(r, t), \\
(g=1,2, \ldots, G)
\end{gathered}
$$

where

$v_{g}$ is the group neutron velocity,
$D_{g}$ is the neutron diffusion coefficient of group $g$,

$\Sigma_{g}^{r}$ is the removal cross-section,

$\Sigma_{g}^{f}$ is the fission cross-section,

$\beta$ is the delayed neutron fraction,

$\chi_{s}^{p}$ is the fraction of the prompt fission neutrons in energy group $g$,

$v_{g}$ is the average number of neutrons released per fission in group $g$,

$\chi_{g}^{d}$ is the fraction of delayed neutrons in group $g$,

$\lambda_{i}$ and $C_{i}$ are the decay constant and concentration of $i^{t h}$ family delayed neutron precursor concentrations, respectively.

The concentration of the delayed neutron precursor also varies with time and follows the differential equation as follows:

$$
\frac{\partial C_{i}(r, t)}{\partial t}=\beta_{i} \sum_{g} v \Sigma_{g}^{f} \phi_{g}(r, t)-\lambda_{i} C_{i}(r, t),
$$

where $\beta_{i}$ is the delayed neutron fraction of the $i^{t h}$ precursor group.

To predict the time dependence of the neutron population, the above sets of coupled equations (equations (1) and (2)) need to be solved. It is extremely difficult to perform a 3D analytical solution, but computational solutions are available with varying degrees of sophistication. The direct method, modal method, nodal expansion method, and flux factorization method are a few of the well known solution methodologies. The flux factorization method that adopts the improved quasistatic (IQS) approximation has been found to be reasonably accurate and easy to implement [2]. In this method, the total neutron flux $\phi$ is factored into a purely time dependent amplitude function $N(t)$ that varies rapidly with time, and is a shape function of space and energy varying slowly with time, $\psi_{g}(r, t)$; that is,

$$
\phi_{g}(r, t)=N(t) \cdot \psi_{g}(r, t)
$$

If this factorization is substituted into equation (1), separate equations for the amplitude factor $N(t)$ and shape function $\psi_{g}(r, t)$ can be derived [5]. For completeness, the final set of equations for the shape function and amplitude factor are included here.

Amplitude factor

$$
\frac{d N}{d t}=\left(\frac{\rho-\beta}{\Lambda}+\frac{k_{0}-1}{k_{0}}\right) N+\sum_{s} \lambda_{s} \eta_{s},
$$

and

$$
\frac{d \eta_{s}}{d t}=\frac{\beta_{s}}{\Lambda} N-\lambda_{s} \eta_{s} \quad(1 \leq s \leq m),
$$


Shape function

$$
v^{-1} \frac{\partial \psi}{\partial t}=\left(-M+F^{P}-\frac{v^{-1} d N}{N d t}\right) \psi+\frac{1}{N} \sum_{s} \chi_{s}^{d} \lambda_{s} C_{s},
$$

where all terms are defined as in the previous equations.

The shape function equation (6) can be solved to in varying levels of approximations. In the simplest approximation, the adiabatic approximation, all time derivatives are removed and the group diffusion equations are solved at several time points based on the instantaneous configuration of the core at the time. The disadvantage is that the effect of the delayed neutrons on the shape function is neglected. However, this is overcome in the more accurate models of quasistatic and improved quasistatic (IQS) schemes. The IQS model is the most accurate model and involves the solution of the full shape function equation without eliminating any time derivatives and thus the results obtained are accurate for slow and fast transients. In the IQS method, the prompt and delayed neutron sources are treated separately. Also, the time derivative of the shape function over the macro interval is approximated using a backward difference scheme. Thus, the factorization method transforms the solving of tedious neutron diffusion equations into solving the amplitude function equations and the shape equation.

Amplitude equations are a set of stiff ordinary differential equations (ODE) that can be solved using any ODE solver capable of handling stiff coupled ODEs. In the present simulation, a modified GRK method, devised and implemented by Sanchez [4], has been used. This method constitutes an algorithm that is easily implemented and provides results with sufficient accuracy for most applications. The main advantage of this method is that it allows systematic time step size control and the estimation of truncation errors is possible at each time step. Shape calculations are performed using the philosophy of the center mesh finite difference approach with triangular meshes. This type of mesh division is suitable for VVER cores in which fuel assemblies are arranged in a hexagonal lattice. Every hexagon is divided into a finite number of triangles and each triangle is considered as one mesh point. Space dependent calculations involve the solution of the shape equation:

$$
-\nabla \cdot D \nabla \psi_{g}+\Sigma_{r_{g}} \psi_{g}=\frac{\chi_{g}}{\lambda} \sum_{g^{\prime}=1}^{G} v_{g^{\prime}} \Sigma_{f, g^{\prime}} \psi_{g^{\prime}}+\sum_{g^{\prime}=1}^{G} \Sigma_{g^{\prime}, g} \psi_{g^{\prime}}
$$

For ease of understanding, the time derivative terms are removed from the shape equations. The solution domain is divided into a grid of regular triangular meshes with a constant pitch. To solve the shape equation, equation (7) is integrated over each triangular region. Within a triangular mesh, the material properties and flux values associated with the mesh cell are assumed to be spatially uniform. The integration of a leakage term over the mesh volume can be converted using the divergence theorem into the summation of the surface or line integrals over the three surfaces of the triangle. Thus, for a triangular mesh ' $i$ ' with volume ' $V$ ' the following can be obtained:

$$
\int_{r_{i}}-\nabla D \nabla \psi d v=\int_{S_{i}}-D \nabla \psi \cdot \hat{n} \cdot d s=\int_{S_{i}} J \cdot \hat{n} d s
$$

The vector $\hat{n}$ is the outward normal to the mesh interfaces. For non-boundary triangles, the neutron current from mesh $i$ to mesh $j, J_{i j}$, can be calculated as follows:

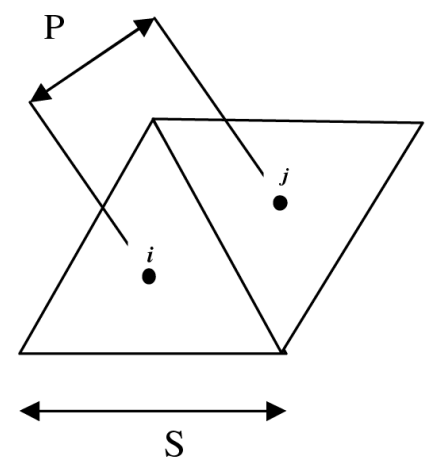

Fig. 1. Triangular Meshes

$$
J_{i j-}=-\left.D_{i}\left(\frac{\partial \psi}{\partial \hat{n}}\right)\right|_{i j}=\frac{-D_{i}\left(\psi_{i j}-\psi_{i}\right)}{(P / 2)}
$$

where $\hat{n}$ is along the line joining $\mathrm{i}$ and $j . P$ is pitch of triangular meshes, and $\psi_{i}, \psi_{j}$, and $\psi_{i j}$ are the flux values at mesh points $i, j$ and the interface, respectively. Similarly, on the other side (inside mesh $j$ ):

$$
J_{i j+}=\frac{-D_{j}\left(\psi_{j}-\psi_{i j}\right)}{(P / 2)}
$$

Then, the current continuity condition, i.e. $J_{i j}+=J_{i j}$, is imposed:

$$
\frac{-D_{i}\left(\psi_{i j}-\psi_{i}\right)}{(P / 2)}=\frac{-D_{j}\left(\psi_{j}-\psi_{i j}\right)}{(P / 2)},
$$

which gives:

$$
\psi_{i j}=\frac{D_{i} \psi_{i}+D_{j} \psi_{j}}{D_{i}+D_{j}}
$$


Substituting $\psi_{i j}$ in any of the current equations:

$$
\begin{gathered}
J_{i j}=J_{i j-}=J_{i j+}=\frac{\bar{D}_{i j}\left(\psi_{i}-\psi_{j}\right)}{P}, \\
\bar{D}_{i j}=\frac{2 D_{i} D_{j}}{D_{i}+D_{j}} .
\end{gathered}
$$

The current $J_{i j}$ is assumed to be constant over the surface $S_{i j}$, which separates meshes $i$ and $j$. In two dimensional geometry, this surface is normally equal to the side (s) of the triangle where $s=\sqrt{3} \mathrm{P}$. Thus, the integration over the $2 \mathrm{D}$ triangular mesh of equation (7) will result in:

$$
\sum_{j} C_{i j}\left(\psi_{i}-\psi_{j}\right)+\Sigma_{r i} \psi_{i} V_{i}=\psi_{i} V_{i}
$$

where

$$
C_{i j}=\frac{\bar{D}_{i j} S_{i j}}{P},
$$

For a 3D problem, the triangular right prismatic meshes are considered. In this case, the integration of the equation (7) over the triangular prism will give:

$\sum_{j} C_{i j}{ }^{H}\left(\psi_{i}-\psi_{j}\right)+\sum_{j} C_{i j}{ }^{V}\left(\psi_{i}-\psi_{j}^{\prime}\right)+\sum_{r i} \psi_{i} V_{i}=\psi_{i} V_{i}(10)$

Here, $j$ is any of the three horizontal neighbors and $j$ ' is any of the two vertical neighbors. $V_{i}$ is the volume of mesh $i . C_{i j}^{H}$ is known as the horizontal coupling kernel and is the same as described in equation (9). In this case, $S_{i j}$ will be the product of a side of the triangle and the height of the triangular prism. The vertical coupling kernel $C_{i j}^{V}$ is given by:

$$
C_{i j^{\prime}}^{V}=\bar{D}_{k k^{\prime}} A_{i},
$$

where

$$
\begin{gathered}
\bar{D}_{k k^{\prime}}=\frac{2 D_{k} D_{k^{\prime}}}{\Delta z_{k} D_{k^{\prime}}+\Delta z_{k^{\prime}} D_{k}}, \\
k^{\prime}=k \pm 1,
\end{gathered}
$$

and $A_{i}$ is the area of the triangular mesh $i . \Delta z_{k}$ and $\Delta z_{k}$, are the axial mesh lengths at the $k^{\text {th }}$ and $k^{\text {th }}$ mesh location. This method has been demonstrated to be sufficiently accurate. The method has been verified and validated against the results of other sophisticated methods such as FEM and NEM [3]. The numerical algorithm of spacetime kinetics is explained in Fig. 2.

\subsection{Boundary Conditions}

Boundary meshes are treated with suitable boundary conditions. Boundary conditions are of the type:

$$
D_{g} \frac{\partial \psi_{g}}{\partial \hat{n}}+\Gamma_{g} \psi_{g}=0
$$

where $\hat{n}$ is the outward normal. $\Gamma_{\mathrm{g}}$ is the ratio of the neutron current to the flux for group $g$. There are many possible boundary conditions that can be applied: that is, a reflective boundary condition $\left(\Gamma_{\mathrm{g}}=0\right)$, a vacuum boundary condition $\left(\Gamma_{\mathrm{g}}=0.4692\right)$, a zero flux boundary condition $\left(\Gamma_{\mathrm{g}}=\infty\right)$, or an albedo boundary condition ( $\Gamma_{\mathrm{g}}$ to be evaluated) [3]. In TRIKIN, provision has been made to handle all kinds of boundary conditions.

\section{THERMAL HYDRAULICS MODEL}

\subsection{Heat Transfer Mechanism}

Heat is generated in the fuel pin through nuclear fission which is then conducted to the clad and finally transferred to the coolant via forced convection. This is shown in Fig. 3. As the radius of the fuel pin is very small compared to with its height, the axial conduction within the fuel pin can be neglected. Additionally, the bulk motion of the coolant is in an axial direction, therefore a 1D axial model of coolant flow is sufficient, i.e. the radial variation of properties within the coolant channel can be neglected. Thus, consistent with the physics of the heat transfer phenomenon, separate equations for the fuel and the coolant can be written.

\subsection{Fuel Heat Conduction}

Fuel heat transfer in the radial pin is governed by a time dependent heat conduction equation. The time dependent radial temperature distribution is governed by the conduction equation in cylindrical geometry:

$$
\frac{1}{r} \frac{\partial}{\partial r}\left[k(T) r \frac{\partial T}{\partial r}\right]+q^{\prime \prime \prime}(t)=\rho(T) C_{p}(T) \frac{\partial T}{\partial t} .
$$

The assumptions involved in this equation are as follows.

1. Axial and azimuthal conduction is ignored.

2. Volumetric heat generation q"' is uniformly distributed over the fuel pellet cross section; gamma heat generation in the gas gap and the cladding is ignored.

3. Thermal conductivity in the fuel pellet and the cladding depends on the temperature, burn up and porosity.

4. Volumetric heat capacity and density in the fuel pellet and cladding are temperature dependent.

5. Thermal conductance in the gas gap is a function of the temperature and burn up. 


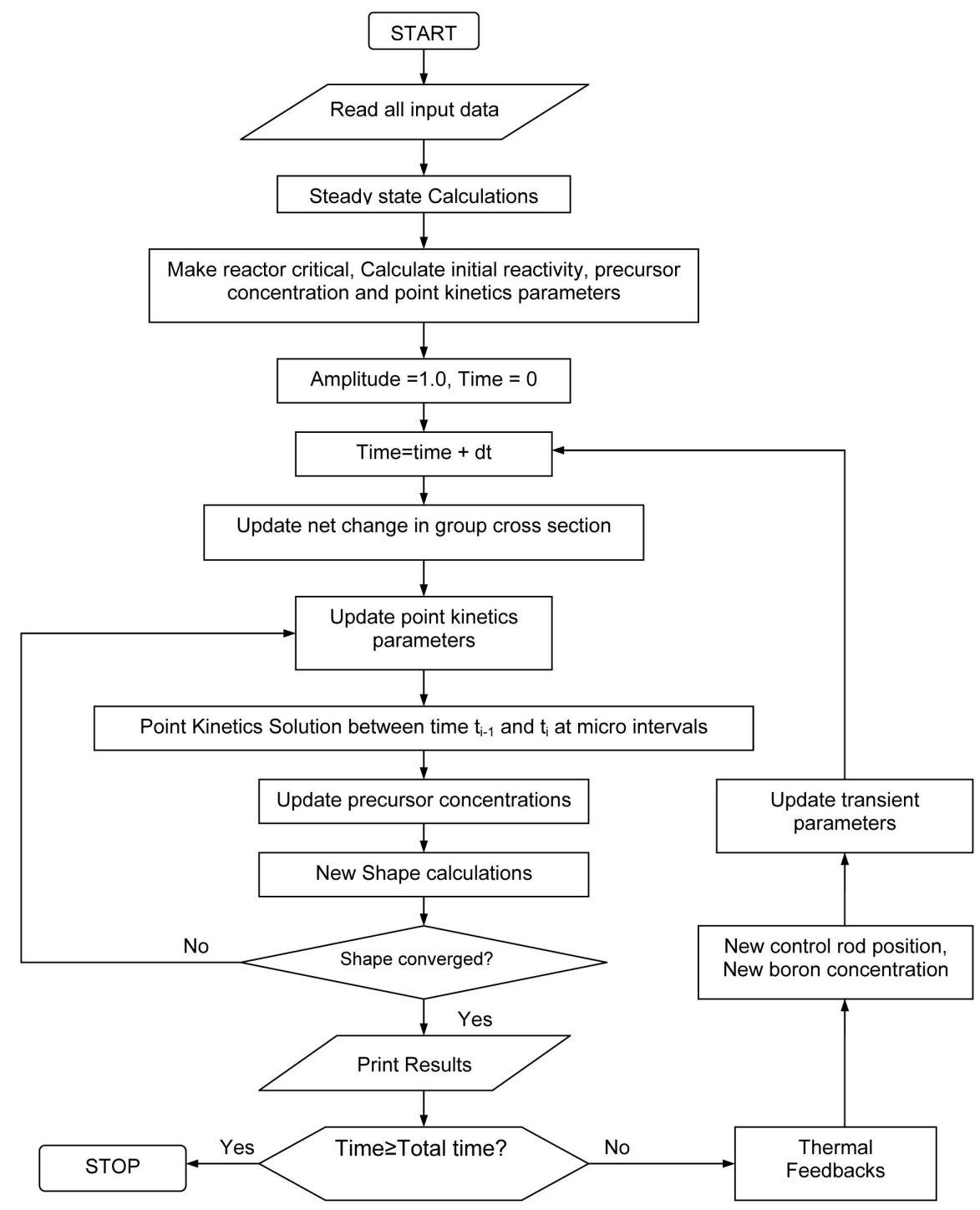

Fig. 2. Computational Flow Chart of TRIKIN Code using IQS Method

The boundary conditions that are applied to equation (12) are:

- The adiabatic boundary condition (condition of zero heat flux) is applied at the inner radius of fuel pin:

$$
-\left.k_{f}(T) \frac{\partial T}{\partial r}\right|_{r_{f i}}=0
$$

- The heat flow across the gap is through conduction, convection and radiation from the fuel surface to the clad. All modes of heat transfer are put together in a single parameter known as the gap conductance $\left(h_{g a p}\right)$, i.e.

$$
-\left.k_{f}(T) \frac{\partial T}{\partial r}\right|_{r_{f o}}=h_{g a p}\left(T_{c i}-T_{f o}\right)
$$

I Fuel pin fission gas plenum

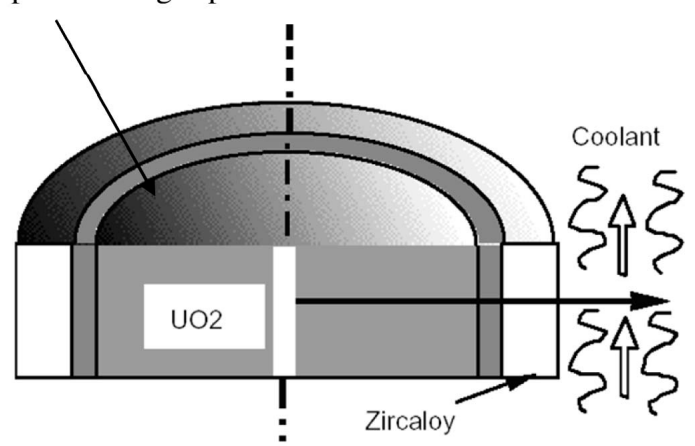

Fig. 3. Heat Transfer Mechanism in a Fuel Pin 
- The heat flow from the clad outer surface is by coolant through forced convection:

$$
-\left.k_{c}(T) \frac{\partial T}{\partial r}\right|_{r_{c o}}=h_{\infty}\left(T_{c o}-T_{\infty}\right)
$$

The heat transfer coefficient $h_{\infty}$ is evaluated from the coolant dynamics calculations iteratively. All explicit, semi implicit or implicit finite difference schemes can be used for the numerical solution of the conduction (parabolic diffusion) equation [6]. In the present model, an implicit scheme is used which is unconditionally stable. An optimal grid in the space and time is determined through numerical experimentation. The numerical experiments showed that 6 to 8 nodes in the fuel pin and a maximum of 2 nodes in the clad are sufficient to maintain the desired level of accuracy in a fuel pin with an order of $1 \mathrm{~cm}$ or less.

\subsection{Coolant Dynamics}

The coolant dynamics phenomenon is modeled by the one dimensional (axial) solution of the mass, momentum and energy equations for a single phase liquid. The coolant properties are assumed to be constant in the channel cross-section, i.e. radial or azimuthal variation in the coolant properties is ignored. In all PWRs, a small radial pressure gradient exists at the core inlet, which vanishes slowly at the core exit and there is no significant radial pressure gradient at the core exit. Thus, compared with the axial pressure drop, which is the driving force for the coolant, the radial pressure drop is very small. This practical feature of PWR allows the radial coolant flow to be ignored in the analysis. In the existing coolant model, the radial coolant motion is ignored and it is assumed that there is no cross flow between the assemblies. This assumption simplifies the coolant problem significantly. Thus, the 3D core thermal hydraulics problem now becomes a problem of multi channels, with channels connected at the inlet and exit plena only. This is shown in Fig. 4.

Solving the mass, momentum and energy conservation equations in each channel will provide the desired coolant properties along the channel. The mass, momentum and

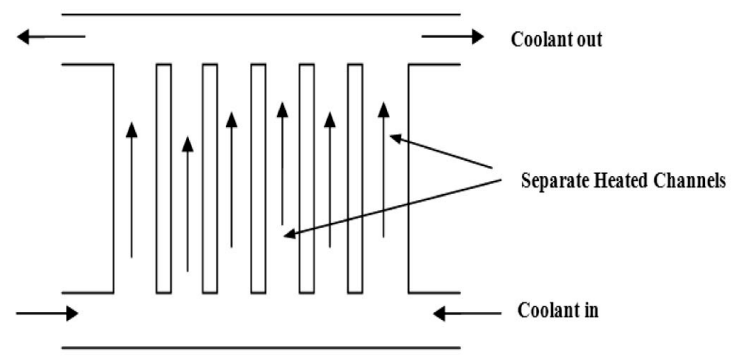

Fig. 4. Core Multi Channel Model energy conservation equations for the single phase fluid in the subcooled portion of the channel are as follows. Continuity (mass conservation):

$$
\frac{\partial\left(\rho A_{Z}\right)}{\partial t}+\frac{\partial\left(G A_{Z}\right)}{\partial z}=0 .
$$

Momentum:

$$
\frac{\partial \dot{m}}{\partial t}+\frac{\partial\left(P A_{Z}\right)}{\partial z}+\left[\frac{\partial}{\partial z}\left(\frac{G^{2}}{\rho} A_{Z}\right)+\rho g A_{z} \cos \theta+\frac{f_{S p} G^{2}}{2 \rho D_{H}} A_{z}\right]=0
$$

Energy:

$$
\frac{\partial\left(\rho H A_{Z}\right)}{\partial t}+\frac{\partial\left(G H A_{Z}\right)}{\partial z}=q_{w}^{\prime \prime} P_{h} .
$$

Other than the spacers, the flow area in the coolant channel is uniform, i.e. $\frac{\partial A z}{\partial z}=0$. The spacers are addressed explicitly in this analysis; therefore, the shrinkage of the flow area near the spacers can be neglected. Further, the incompressible fluid is defined as $\frac{\partial G}{\partial z}=0$. This simplifies the mass and energy equations to:

$$
\begin{gathered}
\frac{\partial \rho}{\partial t}=0 \\
\rho \frac{\partial H}{\partial t}+G \frac{\partial H}{\partial z}=\frac{q_{w}^{\prime \prime} P_{h}}{A_{z}} .
\end{gathered}
$$

The boundary conditions at the clad outer surface may be recalled as:

$$
q_{w}^{\prime \prime}=-\left.k_{c}(T) \frac{\partial T}{\partial r}\right|_{r_{c o}}=h_{\infty}\left(T_{c o}-T_{\infty}\right) .
$$

Substituting this into equation (13) gives:

$$
\rho \frac{\partial H}{\partial t}+G \frac{\partial H}{\partial z}=\gamma h_{\infty}\left(T_{c o}-T_{\infty}\right)
$$

where $\gamma=\frac{P_{h}}{A_{z}}$ is the ratio of the heated perimeter to the cross sectional area of the coolant channel.

The energy equation can be written in temperature form as:

$$
\rho C_{p} \frac{\partial T}{\partial t}+G C_{p} \frac{\partial T}{\partial z}=\gamma h_{\infty}\left(T_{c o}-T_{\infty}\right) .
$$

The engineering parameters, heat transfer coefficient, $h_{\infty}$, and single phase friction factor, $f_{s p}$, can be determined from the empirical correlations. For the heat transfer coefficients, the following dimensionless numbers are defined.

Reynolds Number:

$$
R e=\frac{\rho V D_{h}}{\mu}
$$


where the equivalent channel hydraulic diameter, $D_{h}$, can be determined as:

$$
D_{h}=\left\{\begin{array}{l}
d\left[\frac{4}{\pi}\left(\frac{p}{d}\right)^{2}-1\right] \text { for rectangular lattice } \\
d\left[\frac{2 \sqrt{3}}{\pi}\left(\frac{p}{d}\right)^{2}-1\right] \text { for triangular lattice }
\end{array},\right.
$$

where $P$ is the fuel pin pitch and $d$ is the pin diameter. Prandtl Number:

$$
\operatorname{Pr}=\frac{\mu C_{p}}{k}
$$

Nusselt Number:

$$
N u=\frac{h_{\infty} D_{h}}{k} .
$$

For the fluid, being heated, the commonly used DittusBoelter correlation is used:

$$
N u=0.023 \operatorname{Re}^{0.8} \operatorname{Pr}^{0.4} \text {. }
$$

The single phase friction factor $\left(f_{s p}\right)$ can be determined by correcting the conventional single phase friction factor for circular pipes $\left(f_{s p}^{\prime}\right)$ for triangular or square lattice subchannels. The single phase friction factor for circular pipes $\left(f^{\prime}{ }_{s p}\right)$ is given by:

$$
\begin{aligned}
& f_{s p}^{\prime}=\frac{16}{R e} \text { For } \operatorname{Re} \leq 2000, \\
& f_{s p}^{\prime}=\frac{0.316}{R e^{0.25}} \quad \text { For } 2000<R e \leq 30000, \\
& f_{s p}^{\prime}=\frac{0.184}{R e^{0.20}} \quad \text { For } \operatorname{Re}>30000,
\end{aligned}
$$

for triangular pitch fuel assemblies (in VVER reactors), a correction $f_{c}$ is generally applied, where:

$$
\begin{array}{ll}
f_{c}=1.045+0.071\left(\frac{P}{D}-1\right) & \text { For } \operatorname{Re} \leq 10000 \\
f_{c}=1.036+0.054\left(\frac{P}{D}-1\right) & \text { For } \operatorname{Re}>10000
\end{array}
$$

where $\frac{P}{D}$ is the pitch to diameter ratio of the fuel pin. Thus for triangular pin, single phase friction factor will be:

$$
f_{s p}=f_{s p}^{\prime} * f_{c}
$$

\subsubsection{Pressure Drop across Spacers}

Pressure losses across the spacer grids or wires are form-drag pressure losses that can be calculated using pressure loss coefficients. The spacer pressure drop can be comparable in magnitude to the friction along the bare rod bundle. Rehme [7] has undertaken several studies to determine the pressure drop across spacers with different geometries. After detailed experiments, Rehme devised a list of pressure drop coefficients across the spacers with different geometries and with different arrangements. For triangular arrays and honeycomb type spacers, the pressure drop is given by:

$$
\begin{gathered}
\Delta P=C_{v} \epsilon^{2} \frac{\rho}{2} V_{s}^{2} \\
\epsilon=\frac{A_{s}}{A_{u r}}
\end{gathered}
$$

$C_{v}$ is the modified drag coefficient and its value ranges from 6 to $7 . V_{s}$ is the coolant velocity at a spacer area. $A_{s}$ is the flow area at a spacer region, and $A_{u r}$ is the unrestricted flow area.

\subsubsection{Coolant Thermodynamic Properties}

Light water is used as the moderator and coolant in PWRs. Soluble boron is always present in the coolant for fine reactivity control. The maximum possible boron level in the coolant is not more than $2000 \mathrm{ppm}(0.2 \%)$. Therefore, in the thermal hydraulic analysis, the coolant is considered as pure water and all thermodynamic properties of the coolant are evaluated from the well established empirical correlation used for pure water $[8,9]$.

\subsubsection{Computational Scheme}

The energy equation in temperature form (equation 14) can be descritized using a semi implicit finite differencing scheme as follows:

$$
\begin{gathered}
\bar{\rho} \overline{C_{p}} \frac{\left[\left(\frac{T_{i}^{k+1}+T_{i-1}^{k+1}}{2}\right)-\left(\frac{T_{i}^{k}+T_{i-1}^{k}}{2}\right)\right]}{\Delta t} \\
+\overline{C_{p}} \frac{\left[\left(\frac{G^{k+1} T_{i}^{k+1}+G^{k} T_{i}^{k}}{2}\right)-\left(\frac{G^{k+1} T_{i-1}^{k+1}+G^{k} T_{i-1}^{k}}{2}\right)\right]}{\Delta z} \\
=\frac{\gamma}{2}\left\{\left(h_{\infty i}\left[T_{c o_{i}}-\left(\frac{T_{i}^{k+1}+T_{i}^{k}}{2}\right)\right]\right)+\left(h_{\infty_{i-1}}\left[T_{c o_{i-1}}-\left(\frac{T_{i-1}^{k+1}+T_{i-1}^{k}}{2}\right)\right]\right)\right\}
\end{gathered}
$$

where $\rho$ and $C_{p}$ are the coolant properties at the space and time averaged coolant temperature $T$ and respective nodal pressure.

$$
\begin{gathered}
\bar{T}=\frac{1}{2}\left\{\left[T_{i}^{k}+\frac{\Delta \mathrm{t}^{\mathrm{k}}}{2 \Delta \mathrm{t}^{\mathrm{k}-1}}\left(T_{i}^{k}-T_{i}^{k-1}\right)\right]\right. \\
\left.+\left[T_{i-1}^{k}+\frac{\Delta \mathrm{t}^{\mathrm{k}}}{2 \Delta \mathrm{t}^{\mathrm{k}-1}}\left(T_{i-1}^{k}-T_{i-1}^{k-1}\right)\right]\right\}
\end{gathered}
$$

$T_{c o}$ is iteratively obtained from the solution of the fuel heat 
conduction equation.

To solve the above energy equation (15), some temporal parameters are defined as follows:

$$
\begin{aligned}
& f_{1}=\frac{\bar{\rho} \overline{C_{p}}}{2 \Delta t} \\
& f_{2}=\frac{G^{k+1} \overline{C_{p}}}{2 \Delta z} \\
& f_{3}=\frac{\gamma h_{\infty i}}{4} \\
& f_{4}=\frac{\gamma h_{\infty i-1}}{4} \\
& f_{5}=\frac{G^{k} \overline{C_{p}}}{2 \Delta z} \\
& T_{i}^{k+1}=\frac{T_{i-1}^{k+1}\left(f_{2}-f_{1}-f_{4}\right)+T_{i}^{k}\left(f_{1}-f_{5}-f_{3}\right)}{\left(f_{2}+f_{1}^{k}+f_{3}\right)}\left(f_{1}+f_{5}-f_{4}\right)+T_{c o_{i}}^{k}\left(2 f_{3}\right)+T_{c o_{i-1}}^{k}\left(2 f_{4}\right)
\end{aligned}
$$

This computational scheme is conditionally stable and the condition for numerical stability is [10]:

$$
\begin{gathered}
\Delta t \leq \frac{\Delta z}{|V|} \\
V=\frac{G}{\rho}
\end{gathered}
$$

\subsubsection{Density Evaluation of Two Phase Mixture}

In the transient course, the phase change might occur in some of the peak channels. As the coolant dynamics module is specifically written for a single phase liquid, it cannot address the thermal hydraulics of a two phase coolant. An approximate method [11] is used to determine the average coolant density of the two phase mixture of the heated channel. An assumption is made and the compressibility of the two phase mixture is ignored and the enthalpy content of mixture is determined based on a simple energy balance. This is explained in the following. Consider the energy equation:

$$
\frac{\partial(\rho H)}{\partial t}+\frac{\partial(G H)}{\partial z}=\gamma q_{w}^{\prime \prime}
$$

Ignoring the compressibility of vapor, it can be written as:

$$
\rho \frac{\partial H}{\partial t}+G \frac{\partial H}{\partial z}=\frac{q_{w}^{\prime \prime} P_{h}}{A_{z}}
$$

A mixture enthalpy can be evaluated along the heated channel using the simple finite differencing scheme:

$$
\bar{\rho} \frac{h_{i+1}^{k+1}-h_{i+1}^{k}}{\Delta t}+G \frac{h_{i+1}^{k}-h_{i}^{k}}{\Delta z}=\gamma q_{w}^{\prime \prime}
$$

The quality of steam $(\chi)$ can be evaluated using

$$
h=h_{f}+x h_{f g}
$$

and finally, the quantity of interest and mixture density can be determined from the relation

$$
\rho_{m}=\frac{1}{v_{f}+x v_{f g}}
$$

The wall boundary condition can now be closed using the empirical correlation for the heat transfer coefficient for convection $\left(h_{c}\right)$ and nucleate boiling $\left(h_{N B}\right)$ :

$$
q_{w}^{\prime \prime}=h_{N B}\left(T_{w}-T_{s a t}\right)+h_{c}\left(T_{w}-T_{\infty}\right)
$$

A correction to the frictional pressure drop due to two phase mixture may be applied using the relation:

$$
\phi_{l o}{ }^{2}=1+x\left(\frac{v_{f g}}{v_{f}}\right)
$$

\subsubsection{Boundary Conditions}

Coolant dynamics problems can be solved using many boundary conditions. A simple set of boundary conditions is chosen in which the inlet mass flux, inlet temperature and exit pressure are known. Because the mass flux remains constant along the channel, a single mass velocity approximation is used to solve the momentum equation [10]. These boundary conditions are shown in Fig. 5. A step by step numerical procedure to determine the axial coolant temperature, pressure, and so on is explained here. It is assumed that the converged axial heat flux profile is available from neutronics model for each channel.

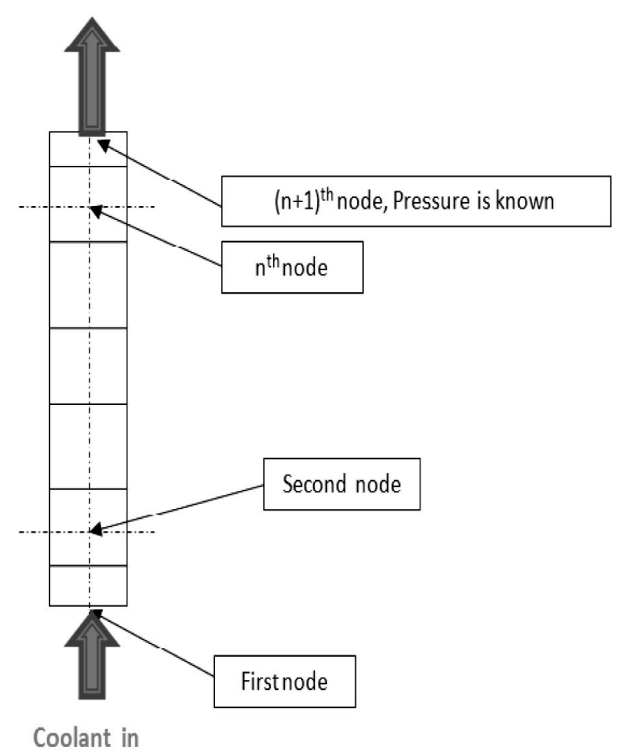

inlet mass flow and inlet temperature is known

Fig. 5. Coolant Channel Boundary Conditions 
1. Solve fuel heat transfer equation to determine fuel and clad temperature along the channel.

2. Start with steady state coolant temperature/density values.

3. Start from the top node and determine the pressure at each node by subtracting all pressure losses.

4. Determine the coolant temperature through equation (16).

5. Obtain the correct mass flux from the momentum equation.

6. Repeat steps 3, 4 and 5. Iterate until the coolant temperature converges to an acceptable limit.

7. Solve the fuel heat transfer equation again to determine the fuel and clad temperatures along the channel.

8. Iterate from step 1 to 7 , until all fuel and coolant temperatures converge.

The computational flow for the thermal hydraulic model is explained in Fig. 6.

The above thermal hydraulic model is coupled to the kinetics model (TRIKIN). The coupling approach is explained in Fig. 7. While coupling two different physics computations, many parameters such as space and time grids, methods of execution and spatial mesh overlays should be considered. Firstly, the same space grid is considered for the core physics and thermal hydraulics computations. The time grid in amplitude calculations of kinetics is based on a prompt neutron lifetime. This time step is optimally evaluated using an adaptive controller. The shape calculations in neutronics are undertaken at larger time steps, which depend on the severity of the transients. The coolant dynamics calculations are performed at time intervals based on the numerical stability criteria. The fuel heat conduction dynamics computations are undertaken at the same time interval as the coolant dynamics as they are unconditionally stable. The core power distribution, coolant density and effective fuel temperature (Doppler temperature) are considered as interface parameters between the thermal hydraulics module and the neutron kinetics module. The smaller the time interval for exchange of interface parameter, the better the accuracy will be, but this situation will increase the computational burden. Thus, an optimal time grid must be chosen.

\section{TRIKIN VALIDATION}

The computational model has been validated against international benchmark problems in VVER reactors. Two problems, AERDYN001 and AERDYN002, have been analyzed. The problems are asymmetric control rod ejection accidents in VVER-440 with and without the Doppler feedback. A horizontal map of the half reactor core is shown in Fig. 8 and the rod that is to be ejected (rod 26) is highlighted.

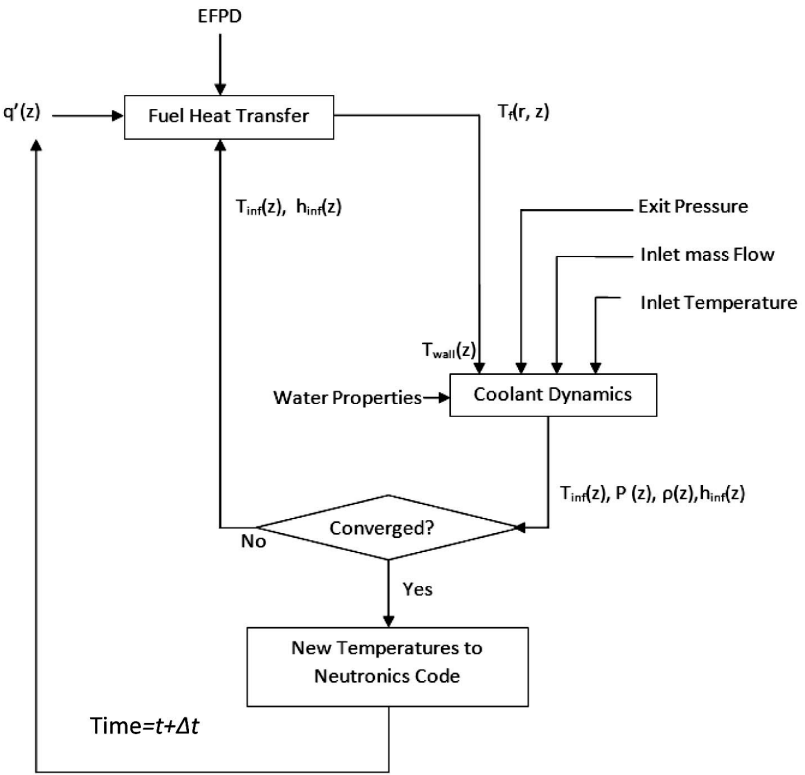

Fig. 6. Computational Scheme for TH Feedback Computations

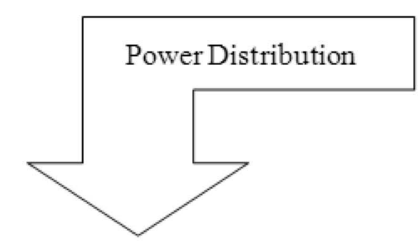

Thermal Hydraulics Coolant density and Fuel Temperature

Fig. 7. Interface Parameters Between Neutronics and Thermal Hydraulics

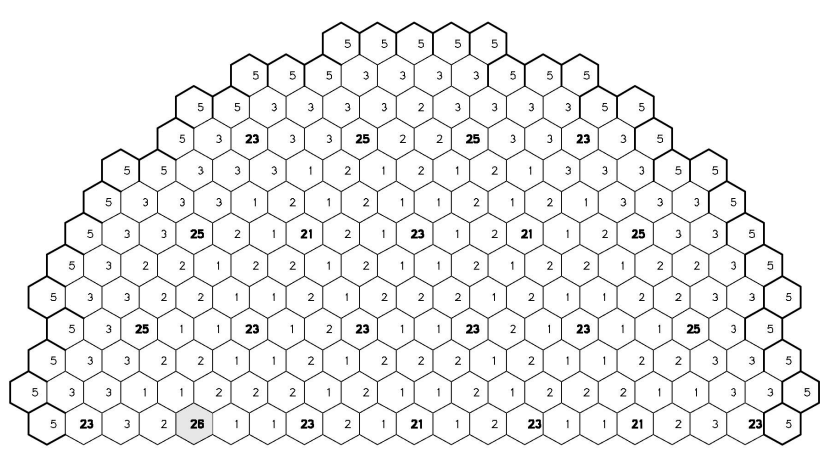

Fig. 8. Horizontal Map of Half Reactor Core (VVER-440 core) 


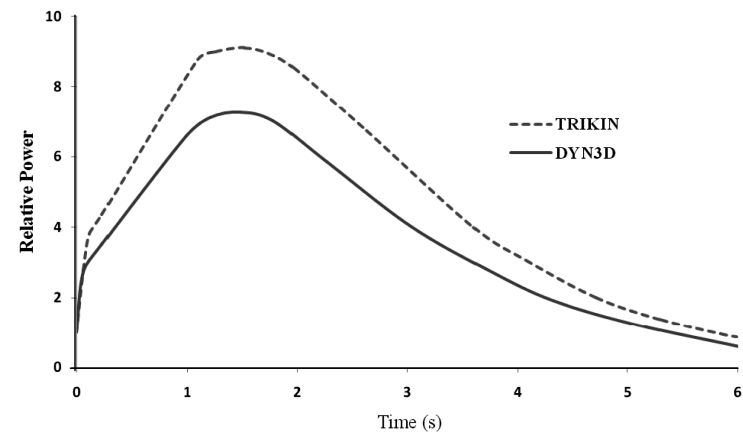

Fig. 9. Normalized Total Power During the Transient

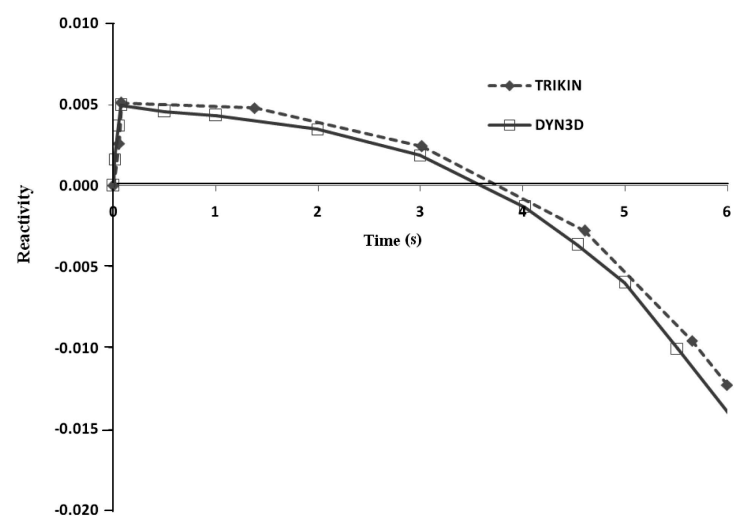

Fig. 10. Reactivity During Transient (TRIKIN)

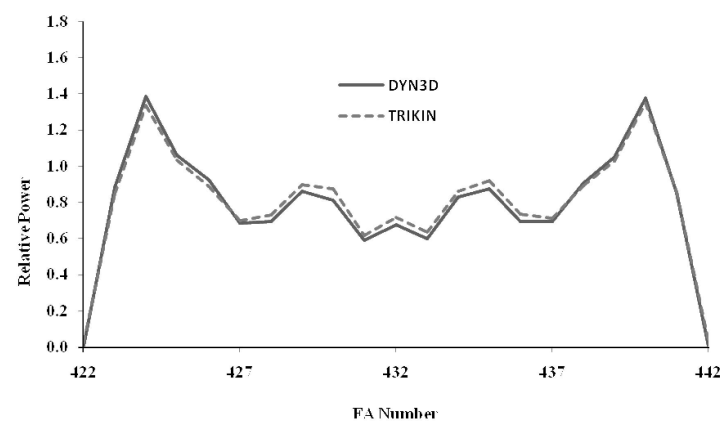

Fig. 11. Normalized Radial Power at Time $=0$

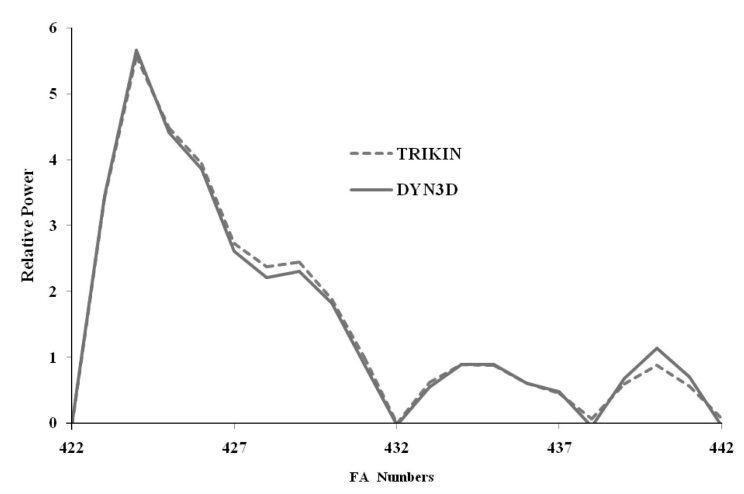

Fig. 12. Normalized Radial Power at 6 Seconds

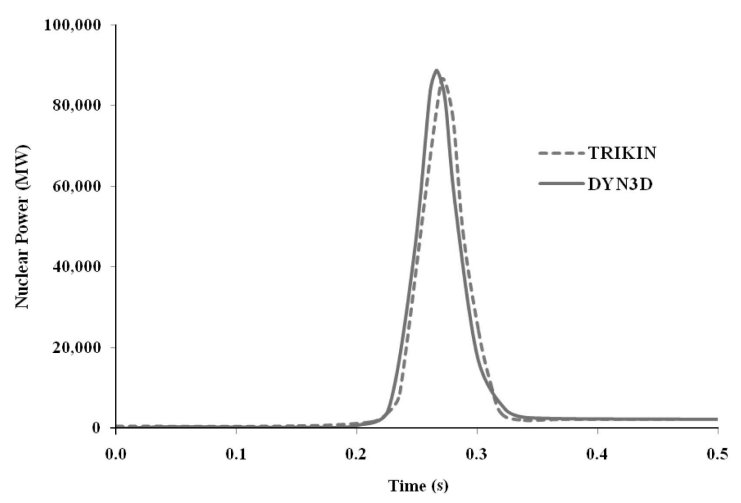

Fig. 13. Nuclear Power

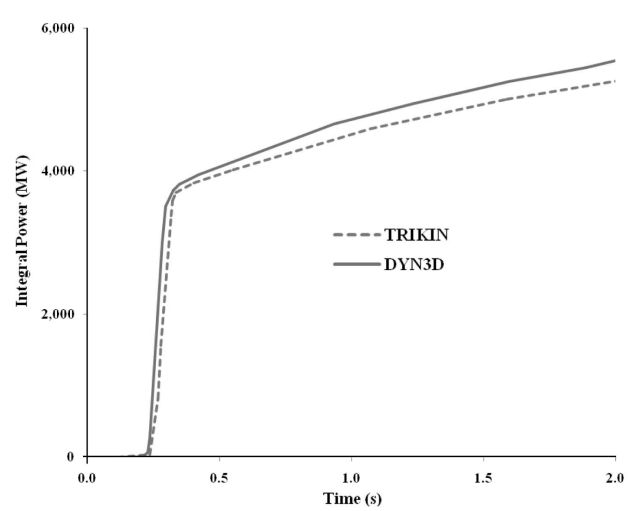

Fig. 14. Integral Power

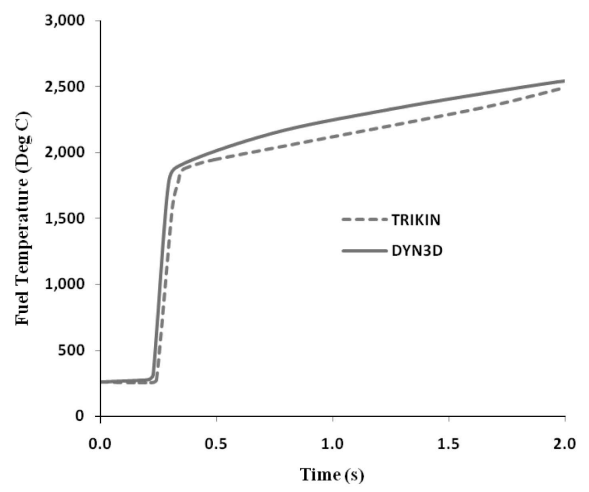

Fig. 15. Fuel Temperature

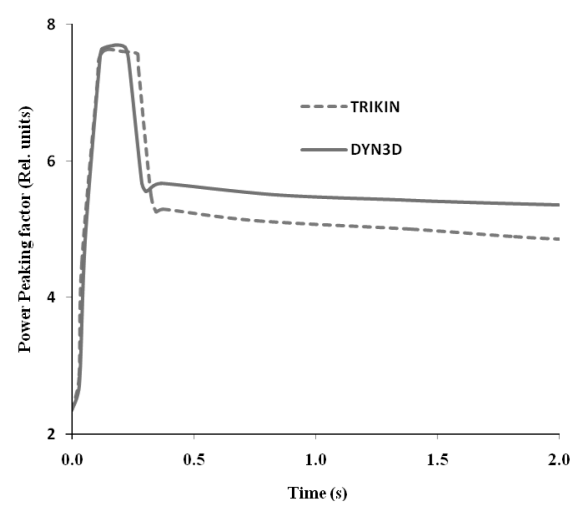

Fig. 16. Power Peaking Factor 


\subsection{Benchmark AERDYN001 [12]}

The transient is analyzed at zero power, so the feedback effects can be neglected. The control rod denoted by number 26 is ejected at $0.08 \mathrm{sec}$. The worth of the ejected rod is marginally below the prompt critical value. The delayed neutron precursors are moving together with the fuel of the absorber followers. Scram is initiated at $1 \mathrm{sec}$ by dropping safety rods 23 and 25 at a constant velocity. These rods take 11 seconds to reach the bottom of the core. The drop of the control rod group 21 is also started at $1 \mathrm{sec}$ with the same velocity. The transient is followed up to $6 \mathrm{sec}$.

In the benchmark, the DYN3D results have been mentioned as reference results; therefore, the TRIKIN results are compared with the DYN3D results. The results are reported in Figs. 9 to12. It has been investigated and demonstrated that each hexagonal FA must be subdivided into 24 triangles for reasonable accuracy [13]. Therefore, in TRIKIN, the core physics computations have been made consistent to a fixed grid involving 24 triangles per FA. These results demonstrate the space-time kinetics capability of TRIKIN.

\subsection{Benchmark AERDYN002 [14]}

This benchmark demonstrates the Doppler feedback (which is the dominant feedback in PWRs) capability of the code. The problem is the simulation of an asymmetric rod ejection accident with Doppler feedback only. Rod 26 (worth approximately two times delayed neutron fraction) is ejected and heat is allowed to accumulate in the fuel. The transient is arrested due to a strong negative Doppler feedback. The results (listed in Figs. 13 to 16) shown can be found to be in good agreement with the DYN3D results.

\section{DISCUSSION}

It can be noticed that the peak power prediction mismatch in benchmark problem AERDYN001 is approximately $12 \%$ (Fig. 9). This is a case of reactivity initiated transients involving a rod worth that is near the prompt critical value. A very small error in the dynamic rod worth (and its peak location in the time domain) may cause a significant deviation in the power predictions. In the present analysis, a very small over prediction $(1.7 \%)$ in the rod value by TRIKIN gives a $12 \%$ rise in the power peak. These deviations (up to $\pm 10 \%$ ) have been observed while analyzing this benchmark using different code systems such as BIPR8, KIKO3D, etc. [12]. Good matching of power profiles (Figs. 11 and 12) demonstrates the excellent core physics capability of TRIKIN. In the benchmark AERDYN002 results, it can be noticed that the power peak and its location is in excellent agreement with DYN3D (Fig. 13). Other parameters such as the integral power (Fig. 14), fuel temperature (Fig. 15), and power peaking factor (Fig. 16), are also in good agreement with the DYN3D results.

\section{SUMMARY}

A new core dynamics code, TRIKIN, has been developed using the available expertise in core physics, kinetics and thermal hydraulics for a reliable safety analysis of VVER reactors. The model uses simple finite differencing schemes in general. The core physics, kinetics and Doppler feedback capability of the code has been demonstrated by validating the code against two benchmark problems, AERDYN001 and AERDYN002, and it has been shown that the results obtained are in good agreement with other code systems. Ongoing efforts incorporate the validation of the complete coupled code, including the coolant and boron feedbacks. Efforts are being made towards making TRIKIN a general code for VVERs to accommodate a vast array of transient problems such as single rod ejection, ejection of a group of rods, inadvertent injection of unborated ECCS water to the coolant, pump switching on, and so on.

\section{ACKNOWLEDGEMENT}

This project has been undertaken with the support of a financial grant from the Atomic Energy Regulatory Board, Mumbai, India. The authors gratefully acknowledge the financial support received to assist in undertaking this project.

\section{REFERENCES}

[1] Advanced Thermal-hydraulic and Neutronic Codes: Current and Future Applications, Summary and Conclusion, OECD/ CSNI Workshop NEA/CSNI/R(2001)9, May 2001.

[2] J. B. Doshi and K. Obaidurrahman, 'Control of Spatial Xenon Oscillations in Large Power reactors', PHYSOR2006, ANS Topical Meeting on Reactor Physics, Vancouver, Canada, September 10-14, 2006.

[3] V. Jagannathan et al., 'Validation of Finite Difference Core Diffusion Calculation Methods with FEM and NEM for VVER-1000 MWe Reactor', PHYSOR-2006, ANS Topical Meeting on Reactor Physics, Vancouver, Canada, Sep. 10-14, 2006.

[4] J. Sanchez, 'On the numerical solution of point reactor equations by Generalized Runge-Kutta methods', Nuclear Science and Engineering, 103, 94-99, 1989.

[ 5 ] D. A. and K. O. Ott, Fast Reactor Kinetics-THE QX1 code, ANL-7769, March 1971.

[6] R. Othman, 'Steady State and Transient Analysis of Heat Conduction in Nuclear Fuel Elements', Master's Degree Project, Stockholm, Sweden 2004.

[ 7 ] K. Rheme, 'Pressure Drop Correlations for Fuel Element Spacers', Nucl. Tech., 17, 15-23, 1973.

[ 8 ] W. J. Garland and B. J. Hand, 'Simple Functions for the Fast Approximation of Light Water Thermodynamic 
Properties', NED, 113, 21-34, 1989.

[9] W. J. Garland et al., 'Extension to the Approximate Functions for the Fast Calculation of Saturated Water Properties', NED, 136, 381-388, 1992.

[10] N. Todreas and M. Kazimi, Nuclear Systems-I, Hemisphere Publishing Corporation, New York, 1990.

[11] J. C. Carter et al., 'SASIA, A Computer code for the Analysis of Fast Reactors', ANL-7607, October 1970.

[12] A. Kereszturi and M. Telbisz, 'A three-dimensional hexagonal kinetic benchmark problem', $2^{\text {nd }}$ AER Symposium, PAKS, Hungary, 1992.

[13] L. Thilagam et al., 'VVER-1000 MOX Core Computational Benchmark analysis using indigenous codes EXCEL, TRIHEX-FA and HEXPIN', Annals of Nuclear Energy 36 1502-1515, 2009.

[14] U. Grundmann and U. Rodhe, 'Definition of second kinetics benchmark of AER', Proceedings of Third Symposium of AER, KFKI AERI, Budapest, 1993. 